Supporting Information

\title{
Facile Peeling Method as a Post-Remedy Strategy for Produceing an Ultrasmooth Self-Assembled Monolayer for High-Performance Organic Transistors
}

Xiaosong Chen ${ }^{\dagger, \S}$, Zeyang $\mathrm{Xu}^{\S}$, Kunjie Wu $\mathrm{Wu}^{\S}$, Suna Zhang ${ }^{\S}$, Hongwei Li ${ }^{\S}$, Yancheng Meng $^{\S}$, Zhongwu Wang ${ }^{\S}$, Liqiang $\mathrm{Li}^{*}, \S$, and Xueming Ma ${ }^{* \dagger}$

*E-mail: 1qli2014@sinano.ac.cn.

*E-mail: xmma@phy.ecnu.edu.cn.

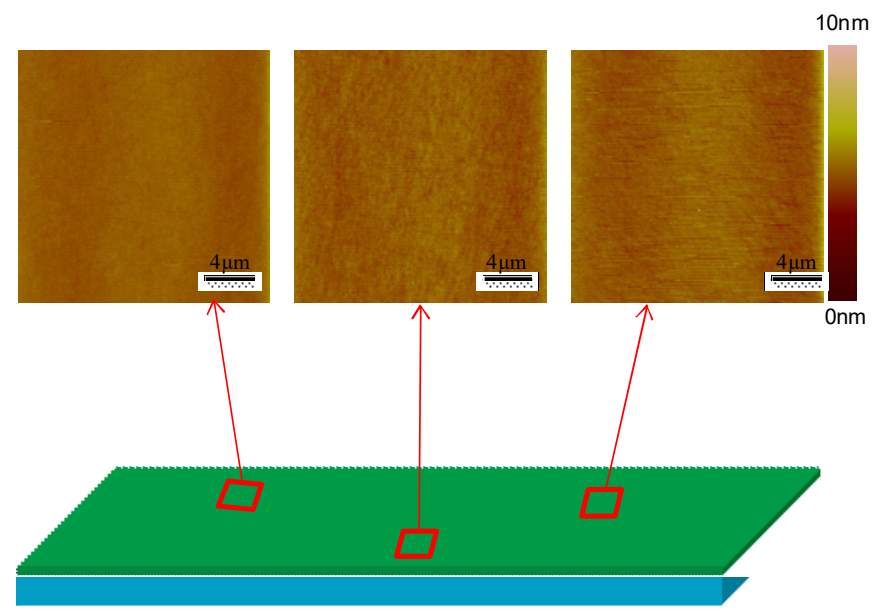

Figure S1. AFM images of OTS monolayers on the different areas. Scan areas are $20 \times$ $20 \mu \mathrm{m}^{2}$ 
a)

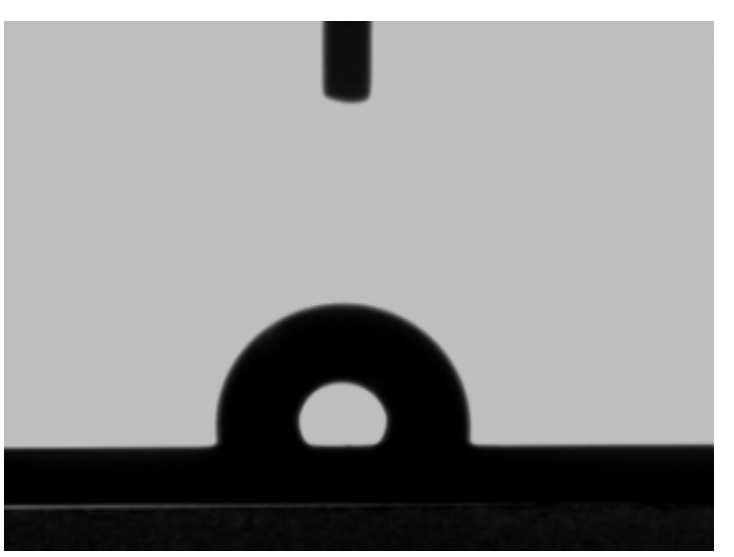

b)

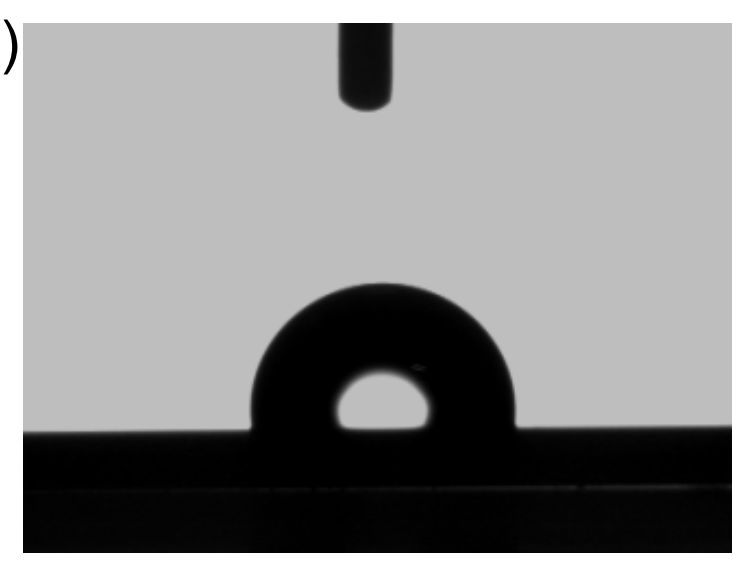

Figure S2. Contact angle of OTS monolayer deposited on $\mathrm{SiO}_{2}$ substrate before (a) and after (b) PMMA peeling treatment. 
a)

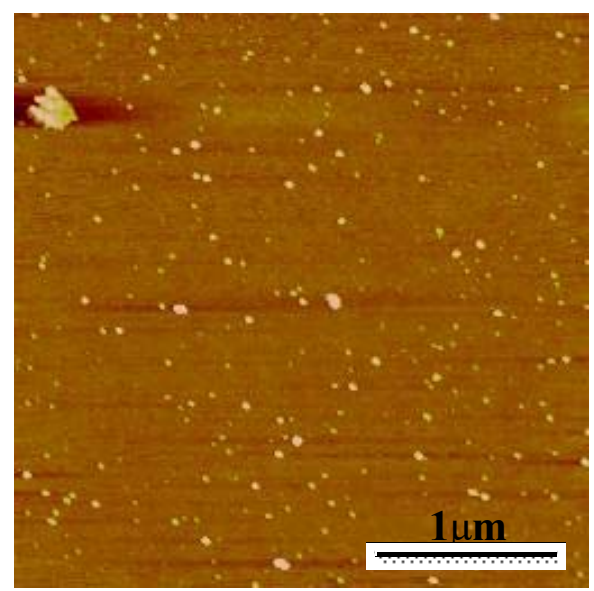

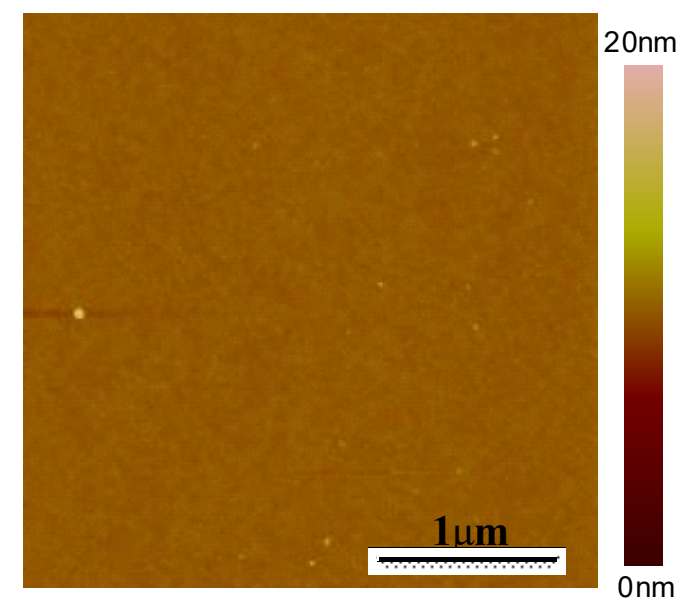

Figure S3. AFM images of $1 \mathrm{H}, 1 \mathrm{H}, 2 \mathrm{H}, 2 \mathrm{H}-\mathrm{Perfluorooctyltriethoxysilane} \mathrm{monolayers}$ before (a) and after (b) PMMA peeling treatment. Scan areas are $3 \times 3 \mu \mathrm{m}^{2}$. 
a)

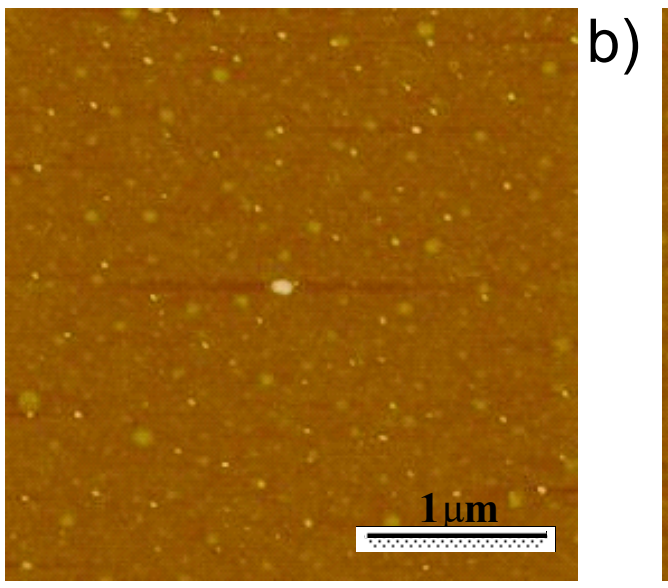

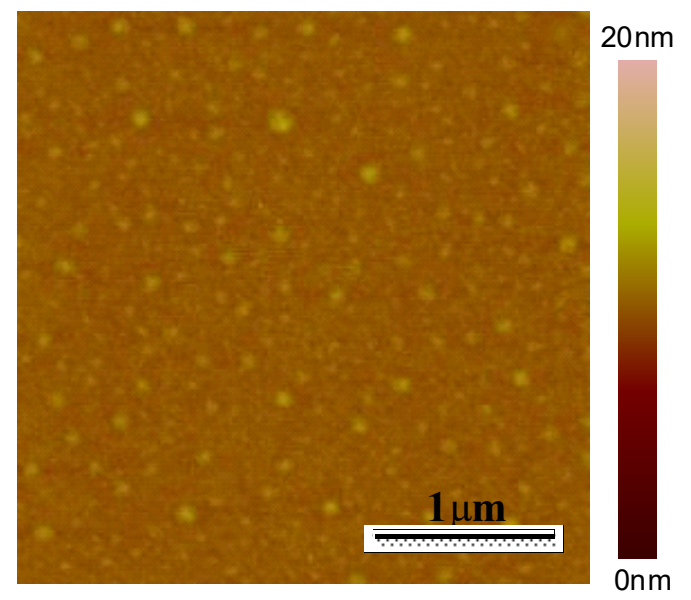

Figure S4. AFM images of APTES monolayers before (a) and after (b) PMMA peeling treatment. Scan areas are $3 \times 3 \mu \mathrm{m}^{2}$. 


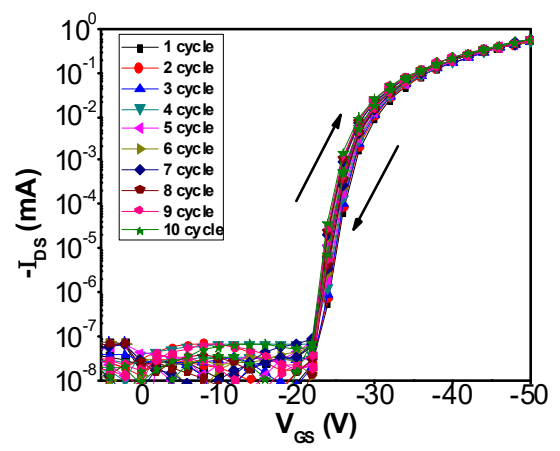

Figure S5. Cyclic test of transfer curves of the DNTT OFETs with ultrasmooth OTS. 

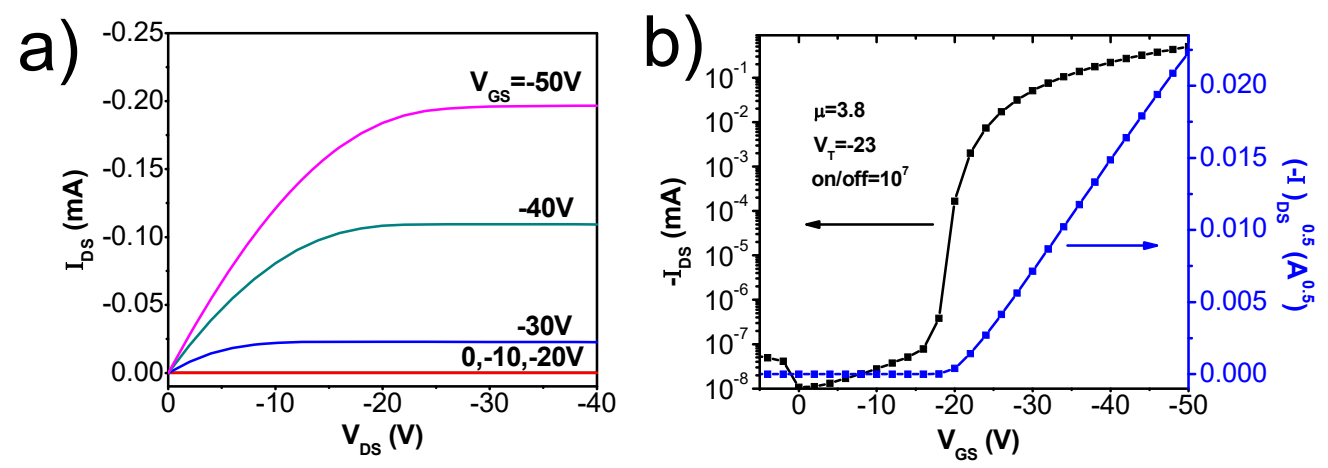

Figure S6. (a) Output and (b) Transfer curves of DNTT OFETs with the pristine OTS (Fig. 2c). 
a)

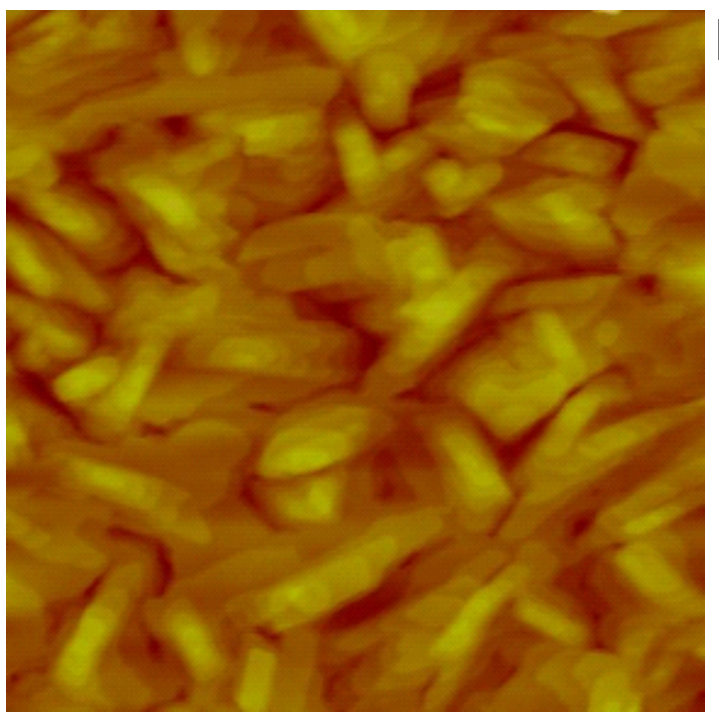

b)

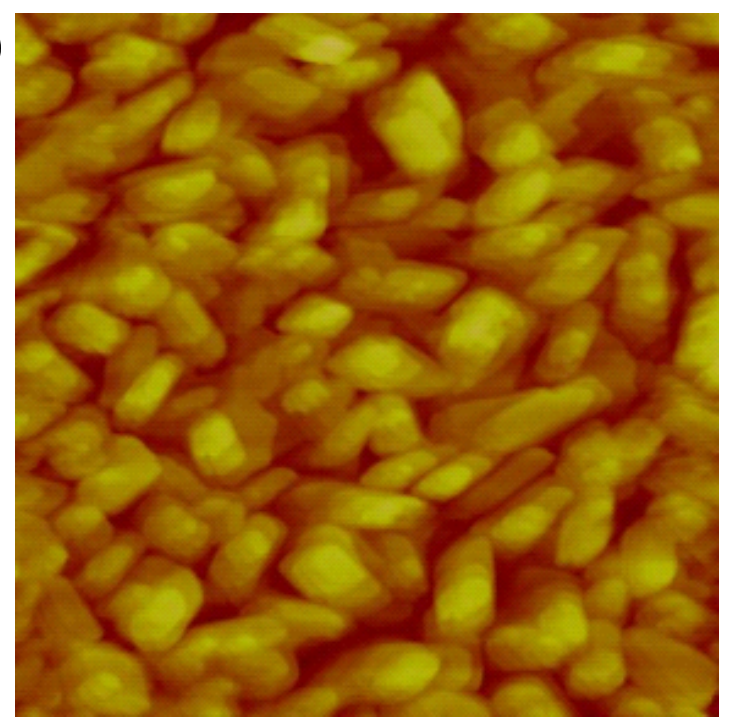

Figure S7. AFM images of PDI film on (a) ultrasmooth OTS with PMMA peeling treatment and (b) pristine OTS without PMMA peeling treatment. Scan areas are $2 \times 2$ $\mu \mathrm{m}^{2}$. 